\title{
Travelling Wave Solutions for Fisher's Equation Using the Extended Homogeneous Balance Method
}

\author{
Mohammad M. Fares ${ }^{1}$, Usama M. Abdelsalam ${ }^{1,2 *}$ and Faiza M. Allehiany ${ }^{3}$
}

${ }^{1}$ Department of Mathematics, University of Technology and Applied Sciences - Al Rustaq, Oman; 'Department of Mathematics, Faculty of Science, Fayoum University, Egypt; ${ }^{3}$ Department of Mathematics, Faculty of Applied Sciences, Umm Al-Qura University, Mecca, Saudi Arabia. *Email: usamaahmad.rus@cas.edu.om.

\begin{abstract}
In this work, the extended homogeneous balance method is used to derive exact solutions of nonlinear evolution equations. With the aid of symbolic computation, many new exact travelling wave solutions have been obtained for Fisher's equation and Burgers-Fisher equation. Fisher's equation has been widely used in studying the population for various systems, especially in biology, while Burgers-Fisher equation has many physical applications such as in gas dynamics and fluid mechanics. The method used can be applied to obtain multiple travelling wave solutions for nonlinear partial differential equations.
\end{abstract}

Keywords: Traveling wave solutions; Partial differential equations; Extended homogeneous balance method and Fisher's equation.

$$
\text { الحلول الموجية لمعادلة فيشر باستخدام طريقة التوازن المتجانس الموسعة }
$$

محمد محمد فارس، أسـامة محمد عبدالسلام و فايزة محمد اللحياني

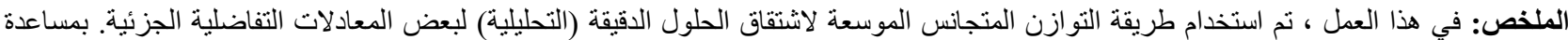

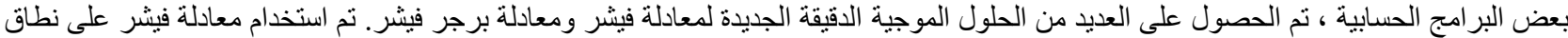

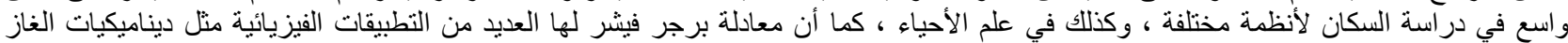

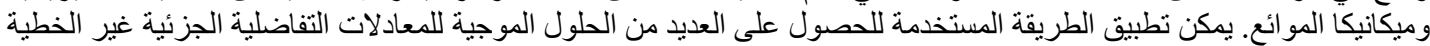
الكلمات المفتاحية: الحلول الموجية ، المعادلات التفاضلية الجزئية ، طريقة التوازن المتجانس الموسعة ، معادلة فيشر. 


\section{TRAVELLING WAVE SOLUTIONS FOR FISHER'S EQUATION}

\section{Introduction}

$\mathbf{T}$

he exact travelling wave solutions of nonlinear evolution equations play an important role in the study of nonlinear physical phenomena, for example, the wave phenomena observed in fluid mechanics, plasma physics, optical fibers, solid state physics, chemical kinematics, chemical physics and geochemistry. Explicit solutions to the mathematical modelling of physical problems are of fundamental importance. Many methods have been developed for finding the exact solutions of nonlinear evolution equations, such as the inverse scattering method [1, 2], bilinear transformation [1, 3, 4], the tanh-function method [5, 6], extended tanh method [7-10], sine-cosine method [11, 12], Fexpansion method [13], general expansion method [14, 15], and $\left(G^{\prime} / G\right)$ method [16-18]. The homogeneous balance (HB) method, which is a direct and effective algebraic method for the computation of exact traveling wave solutions, was first proposed by Wang [19, 20]. Later [21,22], the HB method was extended to search for other kinds of exact solutions. Fan [23] used the HB method to search for Backlund transformation and similarity reduction of nonlinear PDEs. He also showed that there is a close connection among the HB method, Weiss, Tabor, Carnevale (WTC) method and Clarkson, Kruskal (CK) method. The extended homogeneous balance method is used to solve many nonlinear evolution equations [24-28].

The Fisher's equation $[29,30]$ is a nonlinear partial differential equation of second order.

$$
u_{t}=u_{x x}+u(1-u)
$$

Fisher proposed this equation as a model for the propagation of a mutant gene with $u(x, t)$ denoting the density of advantages. This equation is encountered in chemical kinetics, population dynamics, flame propagation, autocatalytic chemical reactions and branching Brownian motion processes. The aim of this work is to propose an extension of the homogeneous balance method to construct more other kinds of exact solutions to nonlinear PDEs. In order to illustrate the effectiveness and convenience of the method, the method is applied to Fisher's equation and Burgers-Fisher equation.

In the following section, let us simply describe the extended homogeneous balance method.

\section{Proposed analytical method}

In general, consider a given PDE, say in two variables

$$
H\left(u, u_{t}, u_{x}, u_{x x}, \ldots\right)=0
$$

We seek for the special solution of Eq. (1), the travelling wave solution, in the form

$$
u(x, t)=u(\zeta), \quad \zeta=x-\lambda t
$$

where $\vartheta$ and $L$ are constants to be determined later. Using the transformation (2), Eq. (1) reduces to a nonlinear ordinary differential equation (ODE). The next crucial step is that the solution we are looking for is expressed in the form

and

$$
u(\zeta)=\sum_{i=0}^{n} a_{i} \omega^{i}+\sum_{i=1}^{n} b_{i}[1+\omega]^{-i}
$$

$$
\omega^{\prime}=k+M \omega+P \omega^{2},
$$

where $a_{i}$ and $b_{i}$ are constants, while $k, M$ and $P$ are parameters to be determined later, $\omega=\omega(\zeta)$, and $\omega^{\prime}=d \omega / d \zeta$. The mechanism for solitary wave solutions to occur is the fact that different effects (such as, the dispersion and nonlinearity) that act to change the wave forms in many nonlinear physical equations have to balance each other. Therefore, one may use the above fact to determine the parameter $n$, which must be a positive integer, and can be found by balancing the highest-order linear term with the nonlinear terms [26]. Substituting (3) and (4) in the relevant ODE will yield a system of ODEs with respect to $a_{0}, a_{i}, b_{i}, k, M, P$ and $\lambda$ (where $i=1, \ldots, m$ ), because all the coefficients of $\omega^{j}$ (where $j=0,1, \ldots$ ) have to vanish. With the aid of MATHEMATICA, one can determine $a_{0}, a_{i}, b_{i}, k$, $M, P$ and $\lambda$.

It is to be noted that the Riccati equation (4) can be solved using the homogeneous balance method as follows

Case I: when $\mathrm{P}=1, \mathrm{M}=0$, the Riccati Eq. (4) has the following solutions

$$
\omega=\{[c] l l-\sqrt{-k} \tanh [\sqrt{-k} \zeta], \text { withk }<0,-\sqrt{-k} \operatorname{coth}[\sqrt{-k} \zeta], \text { with }<0,
$$

$$
\omega=-\frac{1}{\zeta}, \quad \text { with } k=0
$$


and

$$
\omega=\{[c] l l \sqrt{k} \tan [\sqrt{k} \zeta], \quad \text { with } k>0,-\sqrt{k} \cot [\sqrt{k} \zeta], \quad \text { with } k>0 .
$$

Since coth- and cot-type solutions appear in pairs with tanh- and tan-type solutions, respectively, they are omitted in this paper.

Case II:, Let $\omega=\sum_{i=0}^{m} A_{i} \tanh ^{i}\left(p_{1} \zeta\right)$. Balancing $\omega^{\prime}$ with $\omega^{2}$ leads to

$$
\omega=A_{0}+A_{1} \tanh \left(p_{1} \zeta\right) .
$$

Substituting equation (8) into (4), we have the following solution of Eq. (4)

$$
\omega=-\frac{p_{1}}{2 P} \tanh \left(\frac{p_{1}}{2} \zeta\right)-\frac{M}{2 P}, \text { with } P k=\frac{M^{2}-p_{1}^{2}}{4} .
$$

Similarly, let $\omega=\sum_{i=0}^{m} A_{i} \operatorname{coth}^{i}\left(p_{1} \zeta\right)$, then we obtain the following solution:

with $P k=\frac{M^{2}-p_{1}^{2}}{4}$.

$$
\omega=-\frac{p_{1}}{2 P} \operatorname{coth}\left(\frac{p_{1}}{2} \zeta\right)-\frac{M}{2 P}
$$

Case III:, We suppose that the Riccati Eq. (4) has the following solutions of the form

with

$$
\omega=A_{0}+\sum_{i=0}^{m}\left(A_{i} f^{i}+B_{i} f^{i-1} g\right)
$$

$$
f=\frac{1}{\cosh \zeta+r}, \quad g=\frac{\sinh \zeta}{\cosh \zeta+r^{\prime}}
$$

Substituting equations (10) and (11) into (4), we have the following solution of Eq. (4)

$$
\omega=-\frac{1}{2 P}\left(M+\frac{\sinh (\zeta)+\sqrt{r^{2}-1}}{\cosh (\zeta)+r}\right), \text { with } P k=\frac{M^{2}-1}{4}
$$

where $r$ is an arbitrary constant. It should be noticed that solution (12), as $r=1$, degenerates to

$$
\omega=-\frac{1}{2 P}\left[M+\tanh \left(\frac{\zeta}{2}\right)\right]
$$

Case IV:, We suppose that the Riccati Eq. (4) has the following solutions of the form

$$
\omega=A_{0}+\sum_{i=0}^{m} \sinh ^{i-1}\left(A_{i} \sinh \eta+B_{i} \cosh \eta\right)
$$

where $d \eta / d \zeta=\sinh \eta$ or $d \eta / d \zeta=\cosh \eta$ Balancing $\omega^{\prime}$ with $\omega^{2}$ leads to $m=1$

$$
\omega=A_{0}+A_{1} \sinh \eta+B_{1} \cosh \eta .
$$

When $d \eta / d \zeta=\sinh \eta$, we substitute (15) and $d \eta / d \zeta=\sinh \eta$ into (4) and set the coefficient of $\sinh ^{i} \eta \cosh { }^{j} \eta, i=$ $0,1,2, j=0,1$ to zero and solve the obtained set of algebraic equations to get

where $k=\frac{M^{2}-4}{4 P}$, while

$$
A_{0}=\frac{-M}{2 P}, A_{1}=0, B_{1}=\frac{1}{P}
$$

$$
A_{0}=\frac{-M}{2 P}, A_{1}= \pm \sqrt{\frac{1}{2 P}}, B_{1}=\frac{1}{P}
$$

where $k=\frac{M^{2}-1}{4 P}$. To $d \eta / d \zeta=\sinh \eta$ we have

From (16)-(18) we obtain

$$
\sinh \eta=-\operatorname{csesh} \zeta, \cosh \eta=-\operatorname{coth} \zeta .
$$

$$
\omega=-\frac{M+2 \operatorname{coth} \zeta}{2 P} .
$$




\section{TRAVELLING WAVE SOLUTIONS FOR FISHER'S EQUATION}

where $k=\frac{M^{2}-4}{4 P}$, and

where $k=\frac{M^{2}-1}{4 P}$

$$
\omega=-\frac{M \pm \operatorname{csesh} \zeta+\operatorname{coth} \zeta}{2 P} .
$$

\section{Applications of the proposed method} equation.

In this section, we will illustrate the above approach for a class of nonlinear evolution equations namely, Fisher's

\subsection{Example 1. Fisher's equation}

We apply the extended homogeneous balance method to construct the traveling wave solutions for Fisher's equation [24,25]. The Fisher's equation is a nonlinear partial differential equation of second order, of the form

$$
u_{t}=u_{x x}+u(1-u)
$$

Applying the transformation $u(x, t)=U(\zeta), \zeta=x-\lambda t$ to Eq. (21) we find $V$ satisfies the following ordinary differential equation

$$
-U+U^{2}-\lambda U^{\prime}-U^{\prime \prime}=0 .
$$

Balancing $U^{\prime \prime}$ with $U^{2}$ yields $|m|=2$.Therefore, we are looking for the solution in the form

$$
U=a_{0}+b_{0}+a_{1} \omega+b_{1}(1+\omega)^{-1}+a_{2} \omega^{2}+b_{2}(1+\omega)^{-2},
$$

Substituting Eqs. (23) and (4) in Eq. (22), we get a polynomial equation $\omega$. Hence, equating the coefficient of $\omega^{j}$ (i.e., $j=0,1,2, \ldots$ ) to zero and solving the obtained system of overdetermined algebraic equation using symbolic manipulation package MATHEMATICA, results in:

The first set:

$$
\begin{gathered}
a_{1}=0, P \neq 0, a_{0}=\frac{1}{2}\left(-12 P^{2}+12 k P+1\right), b_{1}=\frac{1}{2} \sqrt{3} \sqrt{-384 P^{4}+576 k P^{3}-192 k^{3} P+1}, a_{2}=0, \\
b_{2}=6\left(k^{2}-2 P k+P^{2}\right), \lambda=-480\left(k P^{2} b_{1}-P^{3} b_{1}\right) .
\end{gathered}
$$

The second set:

$$
\begin{gathered}
b_{2}=0, P \neq 0, k=\frac{M^{2}-1}{4 P}, a_{0}=\frac{1}{2}\left(M^{2}+8 k P+1\right), b_{1}=0, a_{2}=6 P^{2}, M \neq 0, \\
=\frac{-36 P M^{6}+432 k P^{2} M^{4}-1728 k^{2} P^{3} M^{2}+211 P M^{2}+2304 k^{3} P^{4}-424 k P^{2}-35 P+70 P a_{0}}{35 M}, \\
\lambda=\frac{6}{7}\left(36 M^{9}-432 k P M^{7}+1728 k^{2} P^{2} M^{5}-73 M^{5}-2304 k^{3} P^{3} M^{3}+160 k P M^{3}+70 a_{0} M^{3}-35 M^{3}\right. \\
\left.-1152 k^{2} P^{2} M+280 k P M-560 k P a_{0} M+2 M+560 k^{2} P a_{1}\right) .
\end{gathered}
$$

For the first set (24), if $M=0, P=1$ we get the solutions satisfying case I for $k>0$. Therefore, the solutions of Fisher's equation of the type (21), will be

$$
\begin{aligned}
& u_{1}(x, t)=a_{0}+\frac{b_{2}+b_{1}(\sqrt{k} \tan (\sqrt{k} \zeta)+1)}{(\sqrt{k} \tan (\sqrt{k} \zeta)+1)^{2}}, \\
& u_{2}(x, t)=a_{0}+\frac{(\sqrt{k} \cot (\sqrt{k} \zeta)+1) b_{1}+b_{2}}{(\sqrt{k} \cot (\sqrt{k} \zeta)+1)^{2}} .
\end{aligned}
$$

For $k<0$,

$$
u_{3}(x, t)=a_{0}+\frac{b_{2}+b_{1}(1-\sqrt{-k} \tanh (\sqrt{-k} \zeta))}{(\sqrt{-k} \tanh (\sqrt{-k} \zeta)-1)^{2}}
$$




$$
u_{4}(x, t)=a_{0}+\frac{(1-\sqrt{k} \operatorname{coth}(\sqrt{k} \zeta)) b_{1}+b_{2}}{(\sqrt{k} \operatorname{coth}(\sqrt{k} \zeta)-1)^{2}}
$$

Now for the solutions satisfying cases II \& III \& IV, we have the compatibility condition,

$$
P k=\frac{M^{2}-p_{1}^{2}}{4} .
$$

Therefore, substitute for $P$ and $k$, from Eq. (24) into Eq. (30) and solve for $p_{1}$. It is found that

$$
p_{1}=-\frac{\sqrt{1-2 a_{0}}}{\sqrt{3}} \quad \text { or } \quad \frac{\sqrt{1-2 a_{0}}}{\sqrt{3}}
$$

Hence, for case II, we get the following solutions:

$$
u_{5}(x, t)=a_{0}+\frac{2 P\left(2 P b_{2}+b_{1}\left(2 P-p_{1}\left(M+2 \tanh \left(\zeta p_{1}\right)\right)\right)\right)}{\left(p_{1}\left(M+2 \tanh \left(\zeta p_{1}\right)\right)-2 P\right)^{2}},
$$

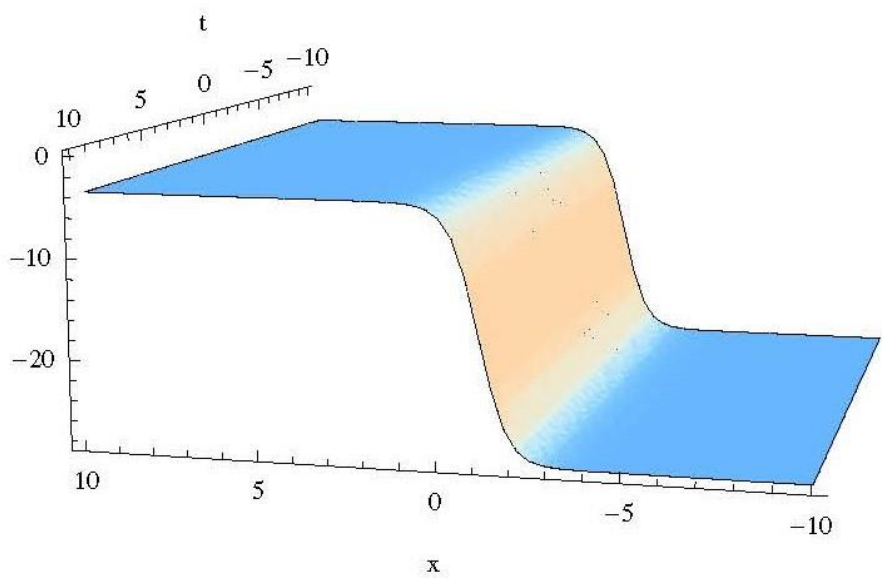

Figure 1. 3D and contour plots of the solution (32) with $a_{0}=-2 P=5$ and $k=5$.

and

$$
u_{6}(x, t)=a_{0}+\frac{2 P\left(2 P\left(b_{1}+b_{2}\right)-\left(M+2 \operatorname{coth}\left(\zeta p_{1}\right)\right) b_{1} p_{1}\right)}{\left(\left(M+2 \operatorname{coth}\left(\zeta p_{1}\right)\right) p_{1}-2 P\right)^{2}},
$$

In the same manner case III, results in the solution

$$
\begin{aligned}
& u_{7}(x, t)=a_{0}+\frac{4 P^{2} b_{2}(r+\cosh (\zeta))^{2}}{\left(M r-2 P r+(M-2 P) \cosh (\zeta)+\sinh (\zeta)+\sqrt{r^{2}-1}\right)^{2}} \\
& -\frac{2 P b_{1}(r+\cosh (\zeta))}{M r-2 P r+(M-2 P) \cosh (\zeta)+\sinh (\zeta)+\sqrt{r^{2}-1}}
\end{aligned}
$$

with the condition that $p_{1}=1$. 


\section{TRAVELLING WAVE SOLUTIONS FOR FISHER'S EQUATION}

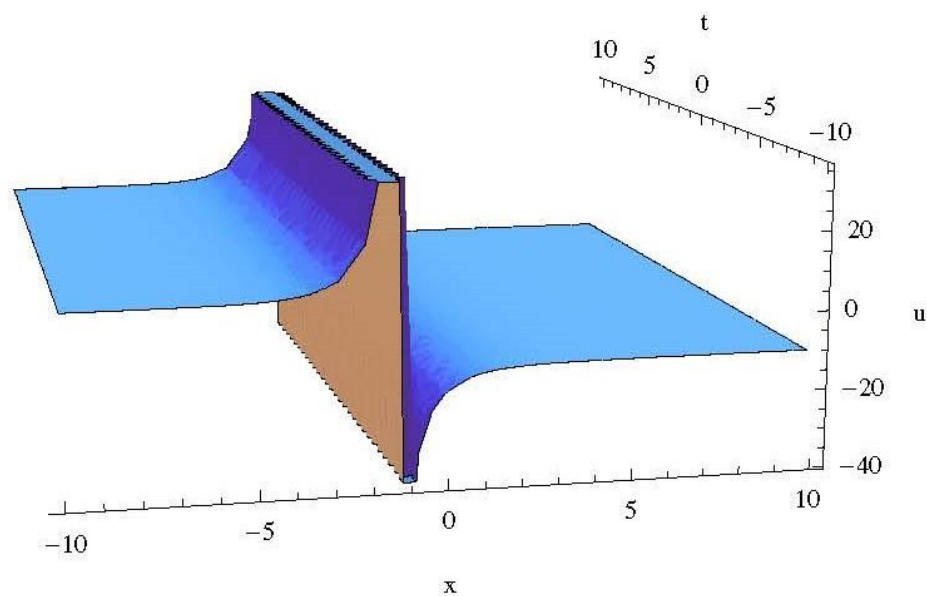

Figure 2. 3D and density plots of the solution (35) with $a_{0}=-2, P=5$ and $k=5$.

For case IV, the solution form is

with the condition that $p_{1}=1$,

$$
u_{8}(x, t)=a_{0}+\frac{2 P\left((M+2 P+\operatorname{coth}(\zeta)+\operatorname{csch}(\zeta)) b_{1}+2 P b_{2}\right)}{(M+2 P+\operatorname{coth}(\zeta)+\operatorname{csch}(\zeta))^{2}}
$$

with the condition that $p_{1}=2$.

$$
u_{9}(x, t)=a_{0}+\frac{2 P\left((-M+2 P-2 \operatorname{coth}(\zeta)) b_{1}+2 P b_{2}\right)}{(M-2 P+2 \operatorname{coth}(\zeta))^{2}}
$$

For the second set we are left only with solutions satisfying cases II \& III \& IV. Since, the main criteria for these cases to be applicable is the compatibility condition,

From (25), it is found that

$$
P k=\frac{M^{2}-p_{1}^{2}}{4} .
$$

$$
p_{1}=1
$$

Therefore, solutions to equation of the type (21), will be

and

$$
u_{10}(x, t)=a_{0}+\frac{3}{2} p_{1}^{2}\left(M+2 \tanh \left(\zeta p_{1}\right)\right)^{2}-\frac{a_{1} p_{1}\left(M+2 \tanh \left(\zeta p_{1}\right)\right)}{2 P}
$$

$$
u_{11}(x, t)=a_{0}+\frac{3}{2}\left(M+2 \operatorname{coth}\left(\zeta p_{1}\right)\right)^{2} p_{1}^{2}-\frac{\left(M+2 \operatorname{coth}\left(\zeta p_{1}\right)\right) a_{1} p_{1}}{2 P}
$$

In the same manner case III, results in the solution

where $p_{1}=1$,

$$
u_{12}(x, t)=a_{0}+\frac{3}{2}\left(M+\frac{\sinh (\zeta)+\sqrt{r^{2}-1}}{r+\cosh (\zeta)}\right)^{2}-\frac{a_{1}\left(M+\frac{\sinh (\zeta)+\sqrt{r^{2}-1}}{r+\cosh (\zeta)}\right)}{2 P}
$$

For case IV, the solution form is

with $p_{1}=1$.

$$
u_{13}(x, t)=\frac{3}{2}(M+\operatorname{coth}(\zeta)+\operatorname{csch}(\zeta))^{2}+\frac{a_{1}(M+\operatorname{coth}(\zeta)+\operatorname{csch}(\zeta))}{2 P}+a_{0}
$$

\subsection{Example 2. Burgers-Fisher equation}

Consider Burgers-Fisher equation [24,25].

$$
u_{t}=u_{x x}+u u_{x}+u(1-u)
$$

Apply the transformation $u(x, t)=U(\zeta), \zeta=x-\lambda t$ to Eq. (43) Then it is reduced to the following ordinary differential equation: 


\section{MOHAMMAD M. FARES ET AL.}

$$
-U+U^{2}-\lambda U^{\prime}+U U^{\prime}-U^{\prime \prime}=0
$$

Balancing $U^{\prime \prime}$ with $U U^{\prime}$ yields $\mathrm{m}=1$. Therefore, we are looking for the solution in the form

$$
U=a_{0}+b_{0}+a_{1} \omega+b_{1}(1+\omega)^{-1}
$$

substituting Eqs. (45) and (4) in Eq. (44), we get a polynomial equation $\omega$. Hence, equating the coefficient of $\omega^{j}$ (i.e., $j=0,1,2, \ldots)$ to zero and solving the obtained system of overdetermined algebraic equations using the symbolic manipulation package MATHEMATICA, results in :

$$
M=2 P+1, k=P+1, a_{1}=0, P \neq 0, b_{1}=\frac{a_{0}}{P}, b_{1} \neq 0, \lambda=-a_{0}+P b_{1}+2
$$

For the first set, as in the previous example, we apply the compatibility condition, in using the solutions satisfying cases II \& III \& IV.

$$
P k=\frac{M^{2}-p_{1}^{2}}{4} .
$$

Therefore, substitute for $P$ and $k$, from Eq. (46), into Eq. (47) and solve for $p_{1}$. It is found that

$$
p_{1}=1 \text { or } p_{1}=-1
$$

Therefore, the solution to the equation of the type (43), will be

and

$$
u_{1}(x, t)=a_{0}\left(1-\frac{1}{1+2 \tanh (x-\lambda t)}\right)
$$

$$
u_{2}(x, t)=a_{0}\left(1-\frac{1}{1+2 \operatorname{coth}(x-\lambda t)}\right)
$$

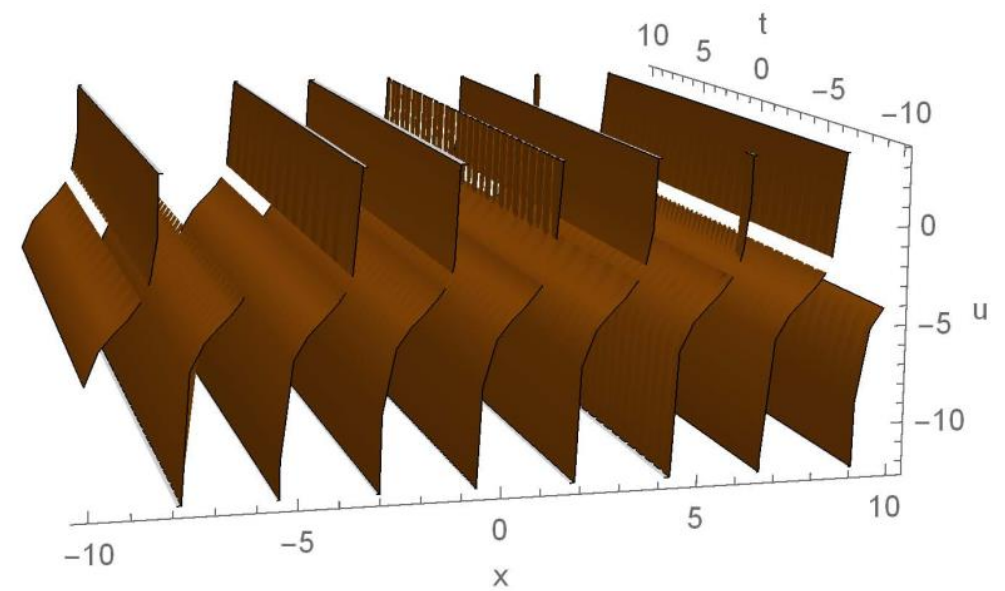

Figure 3. 3D and contour plots of the solution (51) with $a_{0}=0.03 P=1$ and $r=$

In the same manner, case III results in the solution

with the condition that $p_{1}=1$.

$$
u_{3}(x, t)=\frac{\left(-r-\cosh (\zeta)+\sinh (\zeta)+\sqrt{r^{2}-1}\right) a_{0}}{r+\cosh (\zeta)+\sinh (\zeta)+\sqrt{r^{2}-1}}
$$

For case IV, the solution form is

with $p_{1}=1$,

$$
u_{4}(x, t)=\frac{(4 P+\operatorname{coth}(\zeta)+\operatorname{csch}(\zeta)+3) a_{0}}{4 P+\operatorname{coth}(\zeta)+\operatorname{csch}(\zeta)+1}
$$

\section{Conclusion}

In summary, an extended homogeneous balance method with computerized symbolic computation is developed to deal with nonlinear partial differential equations (PDEs). Traveling wave solutions were formally derived for Fisher's equation and Burgers-Fisher equation. This method can be also applied to other nonlinear evolution equations. 


\section{TRAVELLING WAVE SOLUTIONS FOR FISHER'S EQUATION}

\section{Conflict of interest}

The authors declare no conflict of interest.

\section{Acknowledgment}

The authors would like to thank the editor and referees for their valuable comments which helped to improve the manuscript.

\section{References}

1. Drazin, P.G. and Johnson, R.S. Solitons: An Introduction, Cambridge University Press, Cambridge, 1989.

2. Vakhnenko, V.O., Parkes, E.J. and Morrison, A.J. A Backlund transformation and the inverse scattering transform method for the generalised Vakhnenko equation, Chaos Solitons and Fractals. 2003, 17, 683-692.

3. Hirota, R. Direct method of finding exact solutions of nonlinear evolution equations. R. Bullough, P. Caudrey (Eds.), Backlund transformations, Springer, Berlin, 1980, 1157-1175.

4. Lu, D., Hong, B. and Tian, L. Backlund Transformation and N-soliton-like Solutions to the Combined KdVBurgers Equation with Variable Coefficients. International Journal of Nonlinear Science. 2006, 10, 3-10.

5. Malfliet, W. Solitary wave solutions of nonlinear wave equations, American Journal of Physics. 1992, 60, 650654.

6. Gao, Y.T. and Tian, B. Generalized tanh method with symbolic computation and generalized shallow-water waveequation, Computers mathematics with applications. 1997, 33, 115-118.

7. Fan, E. Extended tanh-function method and its applications to nonlinear equations, Physics Letters A. 2000, 277, 212-218.

8. Fan, E. Travelling wave solutions for generalized Hirota-Satsuma coupled KdV systems, Zeitschrift für Naturforschung A. 2001, 56, 312-318.

9. Elwakil, S.A., El-Labany, S.K., Zahran, M.A. and Sabry, R. Exact Travelling wave solutions for the Generalized Shallow water wave equation, Chaos Solitons Fractals. 2003, 17, 121-126.

10. Abdelsalam, U.M. Exact travelling solutions of two coupled (2+1)-Dimensional Equations, Journal of Egyptian Mathematical Society. 25, 125-128, 2017.

11. Wazwaz, A.M. The sine-cosine method for obtaining solutions with compact and noncompact structures, Applied Mathematics and Computation. 2004, 159, 559-576.

12. Wazwaz, A.M. and Helal, M.A. Nonlinear variants of the BBM equation with compact and noncompact physical structures, Chaos Solitons and Fractals. 2005, 26, 67-776.

13. Cai, G., Wang, Q. and Huang, J. A Modified F-expansion Method for Solving Breaking Soliton Equation, International Journal of Nonlinear Science. 2006, 2, 122-128.

14. Sabry, R., Zahran, M.A. and Fan, E. A new generalized expansion method and its application in finding explicit exact solutions for a generalized variable coefficients KdV equation, Physics Letters. A. 2004, 326, 326 : 93.

15. Moslem, W.M., Abdelsalam, U.M. and Sabry, R. and Shukla, P.K. Electrostatic structures associated with multicomponent magnetoplasma with stationary dust particles, New Journal of Physics. 2010, 12, 073010.

16. Wang, M.L., Li, X. and Zhang, J. The $G^{\prime} / G$ - expansion method and evolution erquations in mathematical physics, Physics Letters. A. 2008, 372, 417-421.

17. Abdelsalam, U.M. and Selim, M. Ion-acoustic waves in a degenerate multicomponent magnetoplasma, Journal of Plasma Physics. 2013, 79, 163-168.

18. Selim, M.M. and Abdelsalam, U.M. Propagation of cylindrical acoustic waves in dusty plasma with positive dust. Astrophysics and Space Science, 2004, 353, 535-542.

19. Wang, M.L. Solitrary wave solution for variant Boussinesq equation, Phys. Lett. A. 1995, 199, 169-172.

20. Wang, M.L. Applicatian of homogeneous balance method to exact solutions of nonlinear equation in mathematical physics, Physics Letters A. 1996, 216, 67-75.

21. Fan, E.G. and. Zhang, H.Q. New exact solutions to a system of coupled KdV equations, Physics Letters. A. 1998, 245, 389-392.

22. Yang, L., Zhu, Z. and Wang, Y. Exact Solutions of Nonlinear Equations, Physics Letters A. 1999, $260,55-59$.

23. Fan, E.G. Two new applications of the homogeneous balance method, Physics Letters. A. 2000, 265, 353-357.

24. Abdelsalam, U.M., Allehiany, F.M. and Moslem, W.M. Nonlinear waves in GaAs semiconductor, Acta Physica Polonica A, 2016, 129, 472-477.

25. Abdelsalam, U.M. and Zobaer, M.S. Exact traveling wave solutions of further modified Korteweg De Vries equation in multicomponent plasma. Iranian Journal of Science and Technology, Transactions A 42, pp $2175 \Gamma$ Çô2182 (2017).

26. Abdelsalam, U.M. and Zobaer, M.S. Exact traveling wave solutions of KdV equation for DAWs in superthermal plasma system. Revista Mexicana de Astronomia y Astrofisica 54, pp 363-373 (2018).

27. Abdelsalam, U.M. and Allehiany, F.M. Different Nonlinear Solutions of KP Equation in Dusty Plasmas, Arabian Journal for Science and Engineering 2018, 43(1), 399-406. 
28. Abdelsalam, U.M. and Ghazal, M.G.M. "Analytical Wave Solutions for Foam and KdV-Burgers Equations Using Extended Homogeneous Balance Method", Mathematics 2019, 7(8), 729.

29. Wazwaz, A. The tanh method for generalized forms of nonlinear heat conduction and Burgers-Fisher equations, Applied Mathematics and Computation 2005, 169, 321-338.

30. Wazwaz, A. Analytic study on Burgers, Fisher, Huxley equations and combined forms of these equations, Applied Mathematics and Computation 2008, 195, 754-761.

Received 4 April 2020

Accepted 24 November 2020 